\title{
The tympanic membrane displacement analyser for monitoring intracranial pressure in children
}

\author{
Samson Gwer • Victoria Sheward • Anthony Birch • \\ Robert Marchbanks • Richard Idro • Charles R. Newton • \\ Fenella J. Kirkham • Jean-Pierre Lin • Ming Lim
}

Received: 4 January 2013 / Accepted: 15 January 2013 / Published online: 30 January 2013

(C) The Author(s) 2013. This article is published with open access at Springerlink.com

\begin{abstract}
Purpose Raised intracranial pressure (ICP) is a potentially treatable cause of morbidity and mortality but tools for monitoring are invasive. We sought to investigate the utility of the tympanic membrane displacement (TMD) analyser for noninvasive measurement of ICP in children.

Methods We made TMD observations on normal and acutely comatose children presenting to Kilifi District Hospital (KDH) at the rural coast of Kenya and on children on follow-up for idiopathic intracranial hypertension at Evelina Children's Hospital (ECH), in London, UK.

Results We recruited 63 patients (median age 3.3 (inter-quartile range (IQR) 2.0-4.3) years) at $\mathrm{KDH}$ and 14 children (median age 10 (IQR 5-11) years) at ECH. We observed significantly higher (more negative) TMD measurements in $\mathrm{KDH}$ children presenting with coma compared to normal children seen at the hospital's outpatient department, in both semi-
\end{abstract}

S. Gwer $(\bowtie)$

Department of Medical Physiology, School of Health Sciences,

Kenyatta University, P.O. Box 20880, 00202 Nairobi, Kenya

e-mail:samgwer@gmail.com

S. Gwer

Clinical Research, Afya Research Africa, Nairobi, Kenya

V. Sheward $\cdot$ J.-P. Lin $\cdot$ M. Lim

Paediatric Neurosciences, Evelina Children's Hospital, Guy's and St. Thomas' NHS Foundation Trust, King's Health Partners AHSC, London, UK

\footnotetext{
A. Birch $\cdot$ R. Marchbanks

Neurological Physics Group, Department of Medical Physics and Bioengineering, University Hospital Southampton NHS

Foundation Trust, Southampton, UK

R. Idro $\cdot$ C. R. Newton

Centre For Geographic Medicine Research, Kenya Medical

Research Institute, Kilifi, Kenya
}

recumbent [mean $-61.3(95 \%$ confidence interval $(95 \% \mathrm{CI})$ -93.5 to 29.1$) \mathrm{nl}$ versus mean $-7.1(95 \% \mathrm{CI}-54.0$ to 68.3$) \mathrm{nl}$, respectively; $P=0.03$ ] and recumbent postures [mean -61.4 $(95 \% \mathrm{CI}-93.4$ to -29.3$) \mathrm{nl}, n=59)$ versus mean -25.9 (95\% CI -71.4 to 123.2 ) nl, respectively; $P=0.03$ ]. We also observed higher TMD measurements in ECH children with raised ICP measurements, as indicated by lumbar puncture manometry, compared to those with normal ICP, in both semi-recumbent [mean $-259.3(95 \% \mathrm{CI}-363.8$ to -154.8$) \mathrm{nl}$ versus mean $26.7(95 \% \mathrm{CI}-52.3$ to 105.7$) \mathrm{nl}$, respectively; $P<$ 0.01 ] and recumbent postures [mean $-137.5(95 \% \mathrm{CI}-260.6$ to -14.4$) \mathrm{nl}$ versus mean 96.6 (95\% CI 6.5 to 186.6$) \mathrm{nl}$, respectively; $P<0.01]$.

Conclusion The TMD analyser has a potential utility in monitoring ICP in a variety of clinical circumstances.

Keywords Intracranial pressure · Idiopathic intracranial hypertension $\cdot$ Coma $\cdot$ Child

R. Idro

Department of Paediatrics and Child Health, Mulago Hospital, Makerere University College of Health Sciences,

Kampala, Uganda

\section{R. Newton}

Department of Psychiatry, Oxford University, Oxford, UK

C. R. Newton - F. J. Kirkham

Neurosciences Unit, The Wolfson Centre, Institute of Child Health, University College of London, London, UK

\section{F. J. Kirkham}

Department of Child Health, Southampton University

Hospital NHS Trust, Southampton, UK 


\section{Introduction}

Raised intracranial pressure (ICP) complicates both traumatic and non-traumatic encephalopathies [1-3]. It causes impaired cerebral perfusion leading to brain ischaemia and may result in death due to global ischaemia or herniation of brain tissue [4]. In chronic conditions associated with perturbations of cerebrospinal fluid (CSF) flow dynamics such as idiopathic intracranial hypertension (IIH) [5] and hydrocephalus, it may cause disabling headaches, damage to the optic nerve and inner ear [6] and brain atrophy.

Timely recognition and management of raised ICP may improve outcome [7]. However, the standard tools for monitoring ICP are invasive, require a high level of expertise and have clinically significant risks including haemorrhage, infection and probe displacement [4]. Non-invasive tools could circumvent these limitations, allowing for detailed investigations and life-saving management of diverse raised intracranial pressure syndromes throughout the world.

The tympanic membrane displacement (TMD) analyser (MMS-11 ${ }^{\mathrm{TM}}$, Marchbanks Measurement Systems) was designed for non-invasive monitoring of ICP. It makes use of the communication between the subarachnoid space and the inner ear through the cochlear aqueduct, allowing for transmission of ICP to the perilymphatic space (Fig. 1) [8]. The resulting changes in perilymphatic pressure cause alterations in the kinematics of the middle ear ossicles causing measurable displacements of the tympanic membrane. An air displacement sensor probe sealed into the external auditory meatus detects fluctuations in the volume (in nanolitres) of the external auditory meatus due to these displacements.

Acoustic stimulation of the stapedial reflex with a $1,000-\mathrm{Hz}$ sound stimulus through the ear probe results in contraction of the stapedial muscle, causing movement of the tympanic membrane. The direction and amplitude of movement are determined by the resting position of the stapes footplate within the oval window,

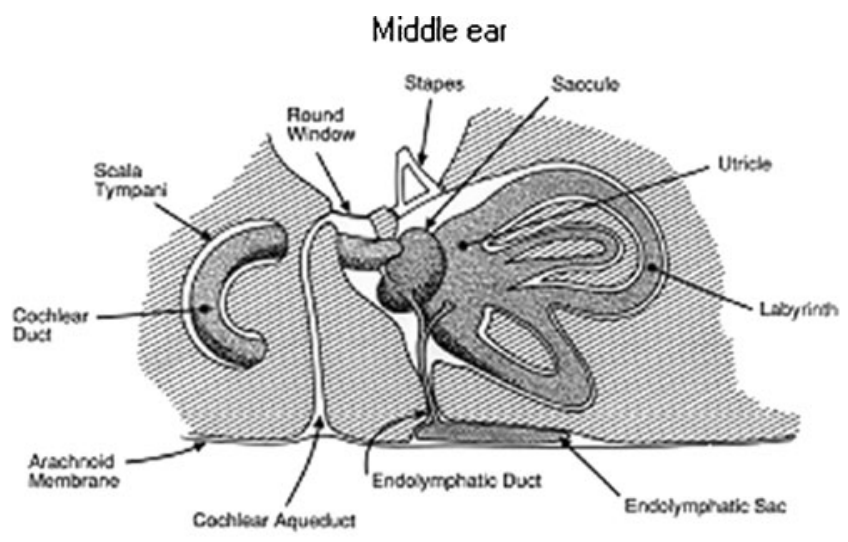

Subarachnoid space

Fig. 1 A schematic representation of the communication between the subarachnoid space and the inner ear through the cochlear aqueduct which is in turn dependent on the perilymphatic fluid pressure and therefore ICP. Raised ICP causes inward motion (negative measurement) of the tympanic membrane, low ICP causes outward motion and normal pressure results in outward or bidirectional motion (Fig. 2). The size of the displacement is quantified by measuring the area under the resulting curve of motion, providing TMD baseline pressure measurements [9].

In this report, we present our experience with TMD baseline pressure measurements in normal and acutely comatose children presenting to Kilifi District Hospital (KDH) at the rural coast of Kenya, and TMD measurements in children followed up for IIH at Evelina Children's Hospital (ECH), in London UK.

\section{Methods}

Setting

We conducted these studies at two sites of different resource status: the paediatric high dependency unit (HDU) and outpatient departments (OPDs) of KDH in Kilifi, a malaria-endemic area in the rural coast of Kenya, and the acute neurology service of ECH in London, UK.

\section{Study population}

At the paediatric HDU of KDH, between November 2007 and September 2009, we evaluated children aged between 6 months and 13 years presenting in acute coma (inability to localise painful stimuli, persisting for longer than $30 \mathrm{~min}$ after correction of hypoglycaemia or treatment of a seizure) [10]. We excluded children known to have sickle cell disease, epilepsy or developmental delay. At the KDH OPD, we evaluated normal children (aged 2 to 13 years) presenting for consideration for recruitment into a study on moderate malnutrition but found not to be eligible because they were well nourished, between August and September 2009. We excluded children who had abnormal middle ear function as determined by tympanometry and those with signs and symptoms of neurological illnesses. The Kilifi studies were approved by the Kenya Medical Research Institute Ethics Committee (SCC no. 1249).

At ECH, we evaluated children aged below 16 years with an established or suspected diagnosis of IIH, with at least 6 months neurological and ophthalmological follow-up. We excluded children with ventriculomegaly, CNS mass lesions or venous sinus thrombosis on neuroimaging. The Evelina study was approved by the Guys and St. Thomas' Research Ethics Committee (ref. 99/09/14).

Standard procedures

The Kenyan children admitted to the HDU were managed according to the standard guidelines for sick children $[11,12]$. 


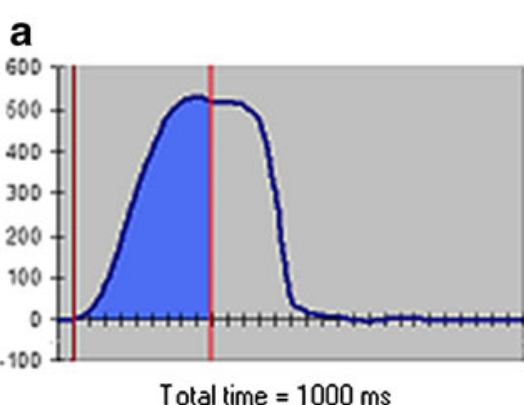

Fig. 2 TMD baseline pressure measurement patterns. a shows a positive deflection of the TMD curve representing a low ICP pressure pattern while $\mathbf{b}$ shows a negative deflection indicating a raised ICP pattern. c A bilateral deflection indicating normal ICP. The area

We classified these children as having cerebral malaria $(\mathrm{CM})$ based on the WHO definition: coma in a child with falciparum malaria parasitaemia in the absence of evidence for an alternative explanation for cause of illness [13]. We considered a diagnosis of acute bacterial meningitis (ABM) when bacteria were isolated on CSF culture, gram staining or bacterial antigen testing, or when there was a CSF leucocyte count of at least 10 per microlitre and the blood-to-CSF glucose ratio was less than 0.67 [14]. Children who had blood culture-confirmed bacteraemia in the absence of any indication of ABM were assumed to have sepsis. We classified children who did not have any history of trauma and no indication of $\mathrm{CM}, \mathrm{ABM}$ or bacteraemia, as unknown encephalopathy. Beyond screening for the study, Kenyan children at OPD did not undergo any extra laboratory or radiological procedures. Children at the $\mathrm{ECH}$ were seen during routine clinic visits for treatment for $\mathrm{IIH}$. Management for $\mathrm{IIH}$ was guided by the standard published guidance $[5,15]$.

\section{Study procedure}

Upon obtaining informed consent from the children's parents or guardians, we carried out tympanometry on the $\mathrm{KDH}$ children. Thereafter, we made TMD measurements on these children in both semi-recumbent (approximately $30^{\circ}$ ) and recumbent postures. If a child became stable, lumbar puncture (LP) manometry was performed.

For ECH children, tympanometry was not done prior to TMD measurements. TMD measurements were similarly done in semi-recumbent and recumbent positions. LP manometry was performed under controlled conditions to standardise for awake carbon dioxide levels as previously described [16], during the TMD observations as part of the management. LP manometry measurements were classified as normal if below $25 \mathrm{~cm}$ of water or raised ICP if at or above $25 \mathrm{~cm}$ of water.

Analysis

We analysed the data using Stata software version 11.0 (StataCorp LP, TX, USA). We explored continuous data

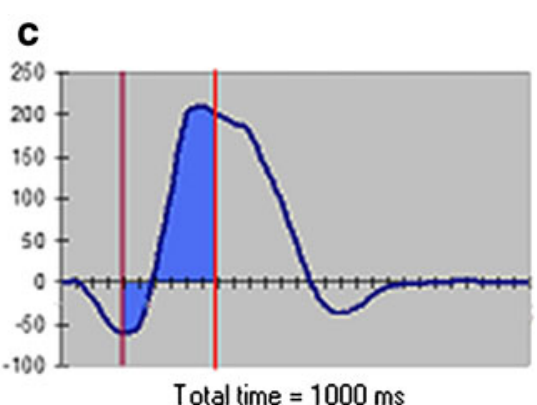

shaded in blue is the area under the curve over the duration of the stapedius reflex response. Calculation of this area is done by the machine software with the user indicating the most negative deflection of the curve and the end of reflex response

graphically using histograms and by applying the ShapiroWilk test to check for violations of the normality assumption. We applied the Student's $t$ test for comparison of normally distributed continuous data and Kruskal-Wallis equality of populations test for non-normal data. We examined for association between categorical data using chi-square and Fisher's exact tests as appropriate. We assessed for statistical significance at the conventional $5 \%$ and where appropriate reported results as odds ratio and $95 \%$ confidence intervals $(95 \% \mathrm{CI})$, or as medians and inter-quartile ranges (IQRs).

\section{Results}

At KDH HDU, we recruited 63 children out of an eligible 113 children (Fig. 3). They had a median age of 3.3 (IQR 2.0-4.3) years and 27 (43\%) were female. Thirty-two had cerebral malaria, 7 bacterial meningitis, 4 sepsis and 20 unknown encephalopathies (Table 1). Eighteen (29\%) of them died and six $(10 \%)$ had gross neurological deficits at the time of

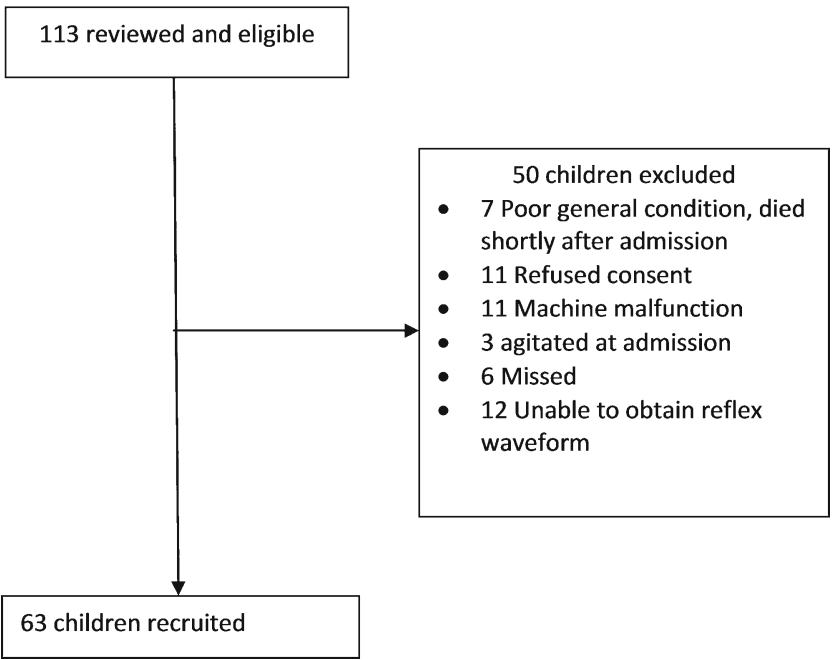

Fig. 3 Study flow chart of patients admitted in coma and recruited at the Kilifi High Dependency Unit. Six patients were eligible for recruitment but the study clinician was not aware at the time of their admission 
discharge. At the KDH OPD, we recruited 24 children. They were of median age 5.6 (IQR 4.6, 6.7) years and 16 were female.

HDU children admitted in coma had higher (more negative) TMD baseline pressure measurements than OPD normal children in both the semi-recumbent $(P=0.03)$ and recumbent postures $(P=0.03)$ (Fig. 4$)$. Among the HDU patients, there were no differences in TMD measurements between those who died and those who survived, in both the semirecumbent [mean $-83.5(95 \% \mathrm{CI}-162.7$ to -4.3$) \mathrm{nl}$ versus mean $-52.4(95 \% \mathrm{CI}-86.6$ to -18.2$) \mathrm{nl}$, respectively; $P=$ 0.39 ] and recumbent postures [mean $-68.4(95 \% \mathrm{CI}-132.2$ to -4.6$) \mathrm{nl}$ versus mean $-58.8(95 \% \mathrm{CI}-97.4$ to -20.2$) \mathrm{nl}$, respectively; $P=0.79]$. Among the survivors, there were no differences in measurements between those who had neurological sequelae and those who survived in both semirecumbent [mean $-35.8(95 \% \mathrm{CI}-82.7$ to 11.0$) \mathrm{nl}$ versus mean $-54.9(95 \% \mathrm{CI}-94.2$ to -15.7$) \mathrm{nl}$, respectively; $P=$ 0.71 ] and recumbent positions [mean $-36.7(95 \% \mathrm{CI}-90.4$ to 17.0) $\mathrm{nl}$ versus mean $-62.3(95 \% \mathrm{CI}-106.9$ to -17.7$) \mathrm{nl}$,

Table 1 Baseline characteristics of Kilifi patients

\begin{tabular}{|c|c|c|}
\hline Characteristic & $\begin{array}{l}\text { Inpatient } \\
(n=63)\end{array}$ & $\begin{array}{l}\text { Outpatient } \\
(n=24)\end{array}$ \\
\hline Age (years) & $3.3(2.0-4.3)$ & $5.6(4.6-6.7)$ \\
\hline \multicolumn{3}{|l|}{ Sex } \\
\hline Male & 36 & 8 \\
\hline Female & 27 & 16 \\
\hline Systolic blood pressure & $98(89-104)$ & - \\
\hline Diastolic blood pressure & $60(50-66)$ & - \\
\hline Axillary temperature & $38.5(37.6-39.0)$ & $37.2(36.7-37.8)$ \\
\hline \multicolumn{3}{|l|}{ Abnormal motor posturing } \\
\hline Opisthotonous & 4 & - \\
\hline Decerebrate & 8 & - \\
\hline Decorticate & 7 & - \\
\hline Papilloedema & 3 & - \\
\hline $\mathrm{pH}$ & $7.39(7.28-7.43)$ & - \\
\hline Partial pressure of $\mathrm{CO}_{2}(\mathrm{kPa})$ & $4.0(3.2-4.8)$ & - \\
\hline Partial pressure of $\mathrm{O}_{2}(\mathrm{kPa})$ & $7.2(5.2-14.9)$ & - \\
\hline Haemoglobin $(\mathrm{g} / \mathrm{dl})$ & $8.7(6.8-10.3)$ & - \\
\hline \multicolumn{3}{|l|}{ Diagnosis } \\
\hline Normal child & - & 24 \\
\hline Cerebral malaria & 32 & - \\
\hline Acute bacterial meningitis & 7 & - \\
\hline Sepsis & 4 & - \\
\hline Unknown encephalopathy & 20 & - \\
\hline Died & 18 & - \\
\hline
\end{tabular}

Summary of the baseline characteristics of the patients recruited at Kilifi District Hospital. Data for inpatients and outpatients were provided side by side for convenience and not for comparison as the two groups of children were different by design

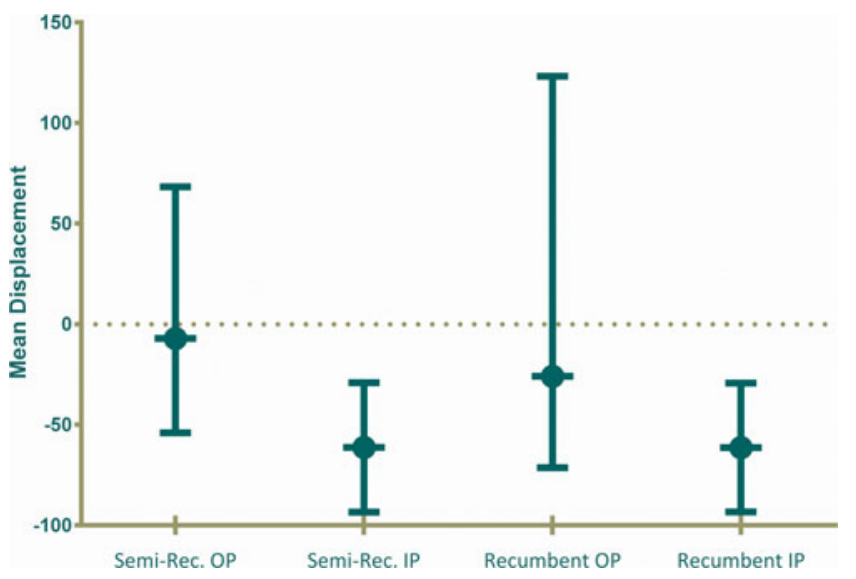

Fig. 4 TMD baseline pressure measurements at Kilifi District Hospital. Semi-recumbent (A) TMD baseline pressure measurements were greater (more negative) for HDU children (inpatient coma) (mean -61.3 (95\% CI -93.5 to -29.1$) \mathrm{nl}, n=63)$ than for OPD normal children (mean $-7.1(95 \%$ CI -54.0 to 68.3 ) nl; $n=24, P=0.03$ ). Recumbent (B) measurements were similarly greater for HDU children (mean $-61.4(95 \% \mathrm{CI}-93.4$ to -29.3$)$ $\mathrm{nl}, n=59)$ than for OPD children (mean $-25.9(95 \% \mathrm{CI}-71.4$ to 123.2$) \mathrm{nl}$; $n=17, P=0.03)$. HDU children showed much less variation with a mean clustered around $-60 \mathrm{nl}$

respectively; $P=0.64]$. No association was noted between TMD measurements and diagnosis, partial pressure of carbon dioxide and haemoglobin level. There was no difference between semi-recumbent and recumbent TMD baseline pressure measurements [mean $-39.7(95 \% \mathrm{CI}-71.8$ to -7.6$) \mathrm{nl}$ compared to mean $-41.8(95 \% \mathrm{CI}-74.7$ to -9.0$) \mathrm{nl}$, respectively; $n=76, P=0.88]$.

Only seven of the HDU children were stable enough to undergo lumbar puncture manometry with corresponding TMD measurements. None of them had an LP manometry measurement above $25 \mathrm{~cm}$ of water (range $5-21.5 \mathrm{~cm}$ of water). The opening LP manometry measurements did not significantly correlate with semi-recumbent TMD measurements $(r=0.49$, $P=0.32)$ or recumbent measurements $(r=0.30, P=0.52)$.

At ECH, we recruited 14 children with headache who were being followed up for IIH (median age 10 (IQR 5-11) years). Five had raised ICP (range $25-65 \mathrm{~cm}$ of water) on initial LP manometry while the rest had lumbar CSF pressure measurements ranging from 6 to $24 \mathrm{~cm}$ of water. We successfully measured TMD in 12 children in the semi-recumbent posture (five with raised ICP) and seven in the recumbent position (two with raised ICP). Semi-recumbent (A) TMD baseline pressure measurements were greater (more negative) for $\mathrm{ECH}$ children with raised ICP (mean -259.3 (95\% CI -363.8 to $-154.8) \mathrm{nl}, n=6$ ) compared to those with normal ICP (mean $26.7(95 \% \mathrm{CI}-52.3$ to 105.7$) \mathrm{nl} ; n=10, P<0.01)$. Recumbent measurements were also similarly greater for children with raised ICP (mean -137.5 (95\% CI -260.6 to -14.4$) \mathrm{nl}, n=4)$ than for those with normal ICP (mean 96.6 (95\% CI 6.5 to 186.6) nl; $n=7, P<0.01$ ) (Fig. 5). There were no significant differences in TMD measurements between normal HDU 
outpatients and ECH patients with normal ICP, for both semirecumbent $(P=0.61)$ and recumbent measurements $(P=0.33)$ (Figs. 4 and 5).

In one ECH patient, follow-up measurements of corresponding TMD and LP manometry measurements were taken. The initial TMD measurement was $-252 \mathrm{nl}$ and the corresponding LP manometer reading was $40 \mathrm{~cm}$ of water. The subsequent TMD measurements were -197 and $-45 \mathrm{nl}$ corresponding to LP manometer readings of 36 and $10 \mathrm{~cm}$ of water, respectively (Fig. 6).

\section{Discussion}

In the Kenyan arm of the study, we observed higher (more negative) TMD measurements in HDU coma patients compared to normal children reviewed at the hospital's OPD. Only seven of the comatose Kenyan children underwent LP manometry, and in all of these, the ICP was less than $25 \mathrm{~cm}$ water. There was no significant correlation between these measurements and TMD measurements. Although there was no significant difference in TMD measurements between HDU patients who survived and those who died, the study was clearly not powered to examine for such differences in clinical outcomes. At ECH, we observed greater negative TMD measurements in patients with raised ICP compared to those with normal ICP. Although measurements of OPD normal Kenyan children were similar to British patients with normal ICP, we do appreciate that these are two different population groups and are not ideal for comparison.

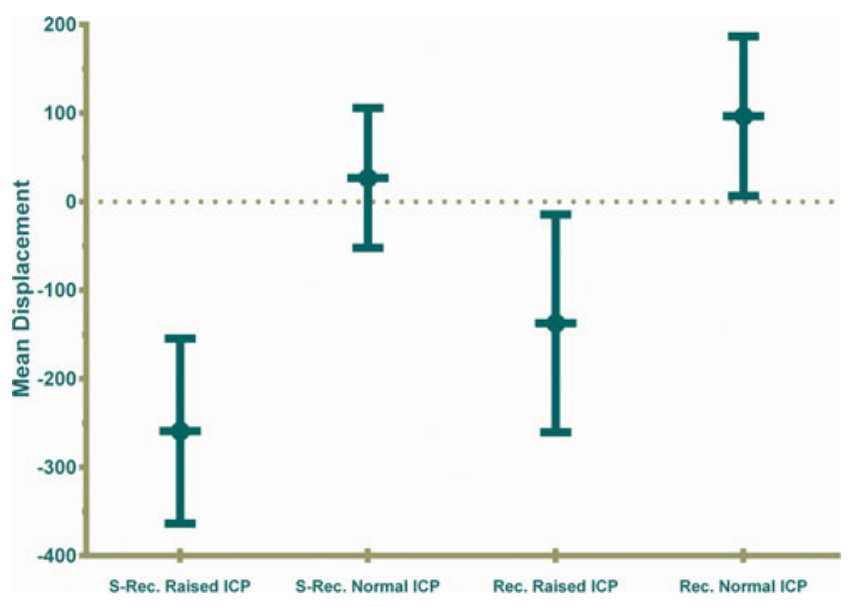

Fig. 5 TMD baseline pressure measurements at Evelina Children's Hospital. Semi-recumbent (A) TMD baseline pressure measurements were greater (more negative) for ECH children with raised ICP (mean -259.3 $(95 \% \mathrm{CI}-363.8$ to -154.8$) \mathrm{nl}, n=6)$ compared to those with normal ICP (mean 26.7 (95\% CI -52.3 to 105.7$) \mathrm{nl} ; n=10, P<0.01)$. Recumbent measurements were also similarly greater for children with raised ICP (mean $-137.5(95 \% \mathrm{CI}-260.6$ to -14.4$), n=4)$ than for those with normal ICP (mean 96.6 (95\% CI 6.5 to 186.6) nl; $n=7, P<0.01$ )

\section{TMD and corresponding LP manometry measurements in patient with IIH}

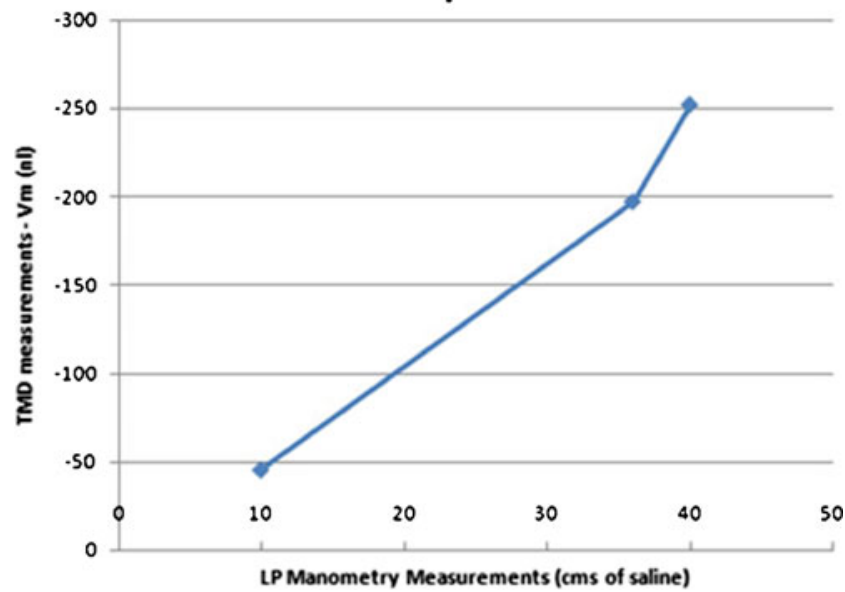

Fig. 6 Comparison between TMD and LP manometry measurements in a 5-year-old child. Corresponding TMD and LP manometry measurements in a 5-year-old child with IIH taken at three separate occasions

Communication between the intracranial space and the inner ear has consistently been demonstrated in a number of studies [8]. The questions are whether the pressure so transmitted is further altered by pressure dynamics and kinematics in the inner and middle ear, perhaps unique in every individual, and which technique is best suited to measure this relationship and provide an accurate index measure of ICP. Previous studies have indicated a significant correlation between baseline TMD and direct ICP measurements in individuals with patent cochlear aqueduct [17-21]. However, there appears to be significant inter-subject variability in this relationship, with consistency in the magnitude of association being observed only within an individual $[19,21]$. In one study, TMD baseline pressure measurements revealed very wide predictive limits of ICP, indicating that TMD measurement could not be used as a reliable surrogate for ICP measurement [19]. Such intersubject variability has been attributed to differences in elasticity of the annular ligaments or in the anatomical relations between the pyramidal eminentia, stapes, stapedial tendon and incudo-stapedial joint [22]. Inter-subject variability could also be due to intracranial anatomical differences which may alter intracranial compliance, perhaps indicating why some individuals appear to suffer clinical symptoms at mildly increased ICP while others are asymptomatic at relatively higher ICP levels. Shimbles et al. (2005) studied a wide patient age range of 4 to 92 years (median of 35 years), whereas our own study was restricted to children of age of 16 years or less [19]. We think that this helps to reduce the inter-subject variability since a number of the above factors are age dependent.

Indeed, in the British arm of our study, we observed correspondence between serial TMD and LP manometry measurements in one individual. We did not demonstrate a 
consistent pattern of correlation between TMD and ICP measurements in both the British and Kenyan arms of the study, in part because many Kenyan children were too ill to have invasive measurements of ICP by conventional lumbar puncture manometry. However, the ability to simply distinguish between patients with raised and normal ICP, and discern those who are severely unwell from those who are normal, suggests a potential utility albeit not yet well understood. It appears possible to have initial concurrent ICP and TMD measurements to define the relationship between the two variables within an individual, before using subsequent TMD measurements to guide management for the individual. It has also been possible to demonstrate pathophysiological patterns in ICP using the TMD analyser and LP on continuous manometry to monitor the continuous relationship between the cardiac cycle arterial pressure and respiratory pressures during positivepressure ventilation which can give a measure of the dynamics of intra-cerebral compliance [23]. However, this was not possible under conditions prevailing at the $\mathrm{KDH}$ HDU and further evaluations within an intensive care unit need to be undertaken.

Our study had a number of limitations. In both arms of the study, the sample sizes involved were too small to enable us make firm conclusions and clearly discern the relationships between TMD measurements, ICP and clinical parameters. The validity of TMD baseline pressure measurements is dependent on normal middle ear function, confirmed using tympanometry [24], which was not consistently done in $\mathrm{ECH}$ patients. When taking measurements on younger children who found lying still over a long duration difficult, it was not always possible to obtain measurements in two different postures. This difficulty would also apply in mildly encephalopathic children of all ages. A smaller headset would enable easier use of the TMD analyser with younger age groups.

Nevertheless, the TMD analyser appears to have a potential utility in ICP or intra-cranial compliance measurement, at a minimum aiding in the simple detection of raised ICP. It could potentially facilitate better monitoring of children with coma in sub-Saharan Africa and other resource poor areas where lack of resources and technical knowhow limit optimal management and outcome of severely ill children. The TMD analyser is convenient and can be used in a variety of other settings, including situations of increased bleeding tendency, increased susceptibility to infection and zero-gravity settings [25]. It could allow for trials in the management of diverse conditions ranging from fluid replacement in diabetic keto-acidosis, cerebral malaria treatment, to management of IIH. With such potential utility in mind, it appears reasonable to conduct more studies, incorporating imaging and other real-time physiological monitoring modalities, include greater patient numbers and examine other modes of TMD measurements.
Acknowledgments We received support from the acute neurology and anaesthetic teams at ECH and the outpatient department team at $\mathrm{KDH}$ who provided help in performing TMD measurements during our studies. The study at KDH was facilitated by Wellcome Trust funding to CN (070114). SG was sponsored by the Royal Society of Tropical Medicine and Hygiene 2009 centenary scholarship to undertake work in the analysis of TMD measurements. This paper is published with permission of the Director of Kenya Medical Research Institute.

Conflict of interest RM is a majority shareholder in Marchbanks Measurement Systems Ltd., a non-profit-making company and spinout from Southampton University that manufactures the TMD Analyser for clinical evaluation purposes. The rest of the authors declare no conflict of interest.

Open Access This article is distributed under the terms of the Creative Commons Attribution License which permits any use, distribution, and reproduction in any medium, provided the original author(s) and the source are credited.

\section{References}

1. Kumar G, Kalita J, Misra UK (2009) Raised intracranial pressure in acute viral encephalitis. Clin Neurol Neurosurg 111:399-406

2. Newton CR, Crawley J, Sowumni A, Waruiru C, Mwangi I, English M, Murphy S, Winstanley PA, Marsh K, Kirkham FJ (1997) Intracranial hypertension in Africans with cerebral malaria. Arch Dis Child 76:219-226

3. Thomale UW, Graetz D, Vajkoczy P, Sarrafzadeh AS (2010) Severe traumatic brain injury in children - a single center experience regarding therapy and long-term outcome. Childs Nerv Syst 26:1563-1573

4. Czosnyka M, Pickard JD (2004) Monitoring and interpretation of intracranial pressure. J Neurol Neurosurg Psychiatry 75:813-821

5. Rangwala LM, Liu GT (2007) Pediatric idiopathic intracranial hypertension. Surv Ophthalmol 52:597-617

6. Weider DJ, Roberts DW, Phillips J (2005) Ventriculoperitoneal shunt as treatment for perilymphatic fistula: a report of six cases. Int Tinnitus J 11:137-145

7. Simma B, Burger R, Falk M, Sacher P, Fanconi S (1998) A prospective, randomized, and controlled study of fluid management in children with severe head injury: lactated Ringer's solution versus hypertonic saline. Crit Care Med 26:1265-1270

8. Traboulsi R, Avan P (2007) Transmission of infrasonic pressure waves from cerebrospinal to intralabyrinthine fluids through the human cochlear aqueduct: non-invasive measurements with otoacoustic emissions. Hear Res 233:30-39

9. Samuel M, Burge DM, Marchbanks RJ (1998) Quantitative assessment of intracranial pressure by the tympanic membrane displacement audiometric technique in children with shunted hydrocephalus. Eur J Pediatr Surg 8:200-207

10. Newton CR, Chokwe T, Schellenberg JA, Winstanley PA, Forster D, Peshu N, Kirkham FJ, Marsh K (1997) Coma scales for children with severe falciparum malaria. Trans R Soc Trop Med Hyg 91:161-165

11. WHO (2005) Pocket book of hospital care for children - guidelines for the management of common illnesses with limited resources. WHO, Geneva

12. Berkley JA, Maitland K, Mwangi I, Ngetsa C, Mwarumba S, Lowe BS, Newton CR, Marsh K, Scott JA, English M (2005) Use of clinical syndromes to target antibiotic prescribing in seriously ill children in malaria endemic area: observational study. BMJ (Clinical research ed) 330: 995

13. WHO (ed) (2010) Guidelines for the Treatment of Malaria 
14. Berkley JA, Mwangi I, Ngetsa CJ, Mwarumba S, Lowe BS, Marsh K, Newton CR (2001) Diagnosis of acute bacterial meningitis in children at a district hospital in sub-Saharan Africa. Lancet 357:1753-1757

15. Soler D, Cox T, Bullock P, Calver DM, Robinson RO (1998) Diagnosis and management of benign intracranial hypertension. Arch Dis Child 78:89-94

16. Lim MJ, Lin JP (2009) The effects of carbon dioxide on measuring cerebral spinal fluid pressure. Childs Nerv Syst 25:783-784

17. Reid A, Marchbanks RJ, Bateman DE, Martin AM, Brightwell AP, Pickard JD (1989) Mean intracranial pressure monitoring by a noninvasive audiological technique: a pilot study. J Neurol Neurosurg Psychiatry 52:610-612

18. Reid A, Marchbanks RJ, Burge DM, Martin AM, Bateman DE, Pickard JD, Brightwell AP (1990) The relationship between intracranial pressure and tympanic membrane displacement. $\mathrm{Br} \mathrm{J}$ Audiol 24:123-129

19. Shimbles S, Dodd C, Banister K, Mendelow AD, Chambers IR (2005) Clinical comparison of tympanic membrane displacement with invasive intracranial pressure measurements. Physiol Meas 26:1085-1092
20. Wagner N, Walsted A (2000) Postural-induced changes in intracranial pressure evaluated non-invasively using the MMS-10 tympanic displacement analyser in healthy volunteers. Acta Otolaryngol Suppl 543:44-47

21. Gosepath K, Maurer J, Amedee R, Mann W (1997) Tympanic membrane displacement analysis after glycerol intake: effects on intracranial and intracochlear fluid pressure. Am J Otol 18:644-647

22. Rosingh HJ, Wit HP, Albers FW (1996) Non-invasive perilymphatic pressure measurement in normal hearing subjects using the MMS-10 tympanic displacement analyser. Acta Otolaryngol 116:382-387

23. Lin J-PR, Eric; Marchbanks, Robert (2005) Non-invasive assessment of intracranial arterial and respiratory pressure waves via the trans aural route in man. Proceedings of the Physiological Society. The Physiological Society, Kings College London

24. Phillips AJ, Marchbanks RJ (1989) Effects of posture and age on tympanic membrane displacement measurements. Br J Audiol 23:279-284

25. Murthy G, Marchbanks RJ, Watenpaugh DE, Meyer JU, Eliashberg $\mathrm{N}$, Hargens AR (1992) Increased intracranial pressure in humans during simulated microgravity. Physiologist 35:S184-S185 